\title{
EL VALOR COMO DISFRUTE DEL CONTRASTE Y LA TEORIAA ACUMULATIVA DEL PROCESO
}

Aparte cualesquiera otras cosas que los filósofos puedan lograr, hay algo que ciertamente deben hacer por nosotros, y ello es mejorar nuestra comprensión de lo que se da a entender por "valor" o "bien". ¿Qué es lo que buscamos cuando buscamos el bien? $O$, más sencillamente, ¿qué es lo que buscamos? Pues el bien es precisamente lo que buscamos, al menos cuando sabemos adecuadamente qué estamos haciendo.

Hace mucho tiempo Platón dio una respuesta a la pregunta. Dijo que el bien era una mezcla de conocimiento, medida o proporción, y placer o satisfacción. Es el término intermedio - la proporción- el que deseo subrayar. ¿Qué significado se le da? Yo sugiero, aunque no sé hasta dónde hubiera estado de acuerdo Platón, que la proporción, en cuanto parte esencial del valor, es armonía, y que la armonía es unidad en la diversidad, similitud en la diferencia, o integración en cuanto incluye elementos contrastantes. Es al mismo tiempo variedad en la unidad y "unidad en la variedad". La unidad en la variedad ha sido durante mucho tiempo una fórmula clásica para la belleza, probablemente la definición de belleza más universalmente aceptada. Decir unidad en la variedad, sin embargo, es sugerir que hay muchos elementos con algo en común. Pero el valor pertenece a la experiencia, y cada experiencia es una. La variedad, pues, debe estar contenida toda ella en la unidad de la experiencia. De esta suerte, si bien indudablemente los factores contrastantes tienen algo en común, tanto la similitud como la diferencia deben estar incluidas dentro de la experiencia singular que tiene el valor. Por consiguiente, la fórmula final parece ser: unidaden-la-variedad-en-la-unidad. El énfasis, así, parece recaer en la unidad. Pero .el asunto no termina aquí, pues si bien cada experiencia es una, hay muchas experiencias. La variedad no se da sólo dentro de las experiencias, sino también entre éstas. Por consiguiente, a menos que pueda haber una experiencia omnicomprensiva, la variedad es en último término una dimensión del valor como unidad. Me propongo argumentar que en un sentido puede haber, y en otro sentido no haber, una experiencia omnicomprensiva. Así, pues, no existe una respuesta simple a la pregunta: ¿Qué es lo primario, la unidad o la variedad?

Históricamente, parece claro que el énfasis se ha puesto casi siempre en la unidad. Si la armonía es la integración de los factores contrastantes, en- 
tonces los filósofos han gritado "integración" a voz en cuello y musitado "factores contrastantes" en un susurro, si es que lo han musitado del todo. Este es el prejuicio filosófico. Yo me propongo impugnar su validez. En la antigua literatura filosófica se nos señala una y otra vez la importancia de la unidad, o "lo uno", en todas sus formas, tales como orden, sistema, permanencia, ley, universalidad; pero, ıqué poco se nos señala la importancia positiva, el valor, de la diversidad, la pluralidad, lo mucho, en sus diversos aspectos, tales como azar, unicidad, novedad, creatividad, particularidad! Los muchos particulares se desprecian como meras apariencias o, a lo sumo, meras ejemplificaciones de lo universal, que es lo único "real en último término". Si "real en último término" significa tener una importancia superior, entonces yo sostengo que aquí nos enfrentamos a una teoría indefensible "en último término". Si la diversidad no unificada no es el bien, tampoco lo es la unidad no diversificada. La variedad es tan necesaria como la unidad, pues ambas son necesarias absolutamente. Así, cada una es tan última como la otra.

En la vida cotidiana los dos aspectos están mejor equilibrados que en los doctos escritos filosóficos. ¿Qué aceptamos como éxito en la búsqueda del valor, o, más sencillamente, como éxito, y qué rechazamos como fracaso? Ciertamente rechazamos la desunión, la discordia, la falta de orden e integridad. Pero, ¿es eso todo? ¿Qué de la unidad "monótona", de la fatigosa repetición de la mismidad, que pueden ser tan ordenadas y sistemáticas como se desee? Así, por ejemplo, un largo poema cuyo esquema de rima sea tal que cada verso deba rimar con todos los demás, será perfectamente ordenado en su rima, pero no será aceptable. Tampoco habrá nada desordenado en el programa de un concierto que consista en la alternación de dos canciones una y otra vez, pero no será un buen programa. Sin embargo, la repetición monótona no es la única forma de fracaso relativo, de no-bondad. Si se ofreciera una sucesión de dos notas musicales contrastantes, en relación armoniosa entre sí, como una composición completa, se objetaría que tal pauta musical es trivial. Los niveles superiores del valor implican una mayor complejidad, una mayor riqueza de contrastes y de contrastes entre contrastes, una mayor variedad en los tipos de variedad que los niveles inferiores. El bien, en realidad, se mide de tres maneras: primera, según el logro de la unidad adecuada a la variedad integrada; segunda, según el logro de la diversidad adecuada a la unidad; y tercera, según el nivel de complejidad. Estas tres maneras son distinguibles. El fracaso en el logro de la unidad adecuada se llama discordia o conflicto; el fracaso en el logro de la diversidad adecuada se llama monotonía; el fracaso en el logro de un nivel de complejidad unificada tan alto como se considere posible, se llama "trivialidad" (algunas veces, "puerilidad"). Dos notas en relación armónica no están no-unificadas, ni son indebidamente repetitivas. Pero no son de gran valor en sí mismas, por ser demasiado simples. 
También podemos expresar estos principios de la siguiente manera. En cada nivel de complejidad puede haber una relación equilibrada o desequilibrada entre la similitud y la diferencia de los elementos. El mal es, o bien un equilibrio que favorece la variedad, es decir, discordia, o bien un equilibrio que favorece la unidad, es decir, monotonía. Pero el eludir ambos defectos no es aún necesariamente un alto nivel de éxito; es un bien, pero puede no ser un gran bien, pues, dados sólo unos pocos elementos, la armonía es trivial, pueril o subhumana.

Veamos un ejemplo. Considérese un animal salvaje que ha sido encerrado en una jaula. El resultado inmediato para el animal es la discordia, pues él desea correr libremente según su costumbre, y las rejas se lo impiden. Supóngase, sin embargo, que al cabo de cierto tiempo el animal acepta las condiciones del encierro. Entonces ya no hay conflicto, cuando menos no con la misma o parecida intensidad. La unidad de experiencia ha sido recobrada. Pero, ¿es ésta una unidad de elementos adecuadamente diversificados? Seguramente ya existe cierta monotonía y aburrimiento. Considérese entonces al animal fuera de la jaula. Su vida está bien unificada en su mayor parte, puesto que sus impulsos están controlados armoniosamente por el instinto y el hábito; pero los cambios en el medio ambiente y las necesidades de hallar su alimento y de escapar a sus enemigos proporcionan una variedad de estímulos suficiente para evitar la monotonía. Semejante criatura está bien equilibrada, en lo principal, entre la unidad y la diversidad. Es, en otras palabras, un animal saludable en su medio ambiente normal y, por tanto, adecuado. Pero en comparación con un orden superior de criatura, como es el hombre, o quizá uno de los grandes simios, puede presumirse que los valores que pueden lograr los animales inferiores son algo triviales, pues ciertos órdenes de contraste que se encuentran en los niveles superiores de existencia están ausentes en los inferiores. De esta suerte, el largo alcance de la memoria del hombre proporciona al trasfondo de su conciencia una complejidad tal, que, comparada con ella, cualquier cosa que sea posible para una ostra o un insecto - - incluso para un chimpancé- debe ser enormemente más simple.

La capacidad para el pensamiento abstracto, que culmina en lo que llamamos conciencia, en contraste con el mero sentimiento, significa dimensiones de contraste que de otra suerte estarían ausentes. No significa mayor unidad, en el sentido de mayor libertad respecto de la discordia, pues una "ostra satisfecha" difícilmente puede ser superada en este respecto (es de suponerse) por un budista en el Nirvana. Los organismos inferiores no están menos bien integrados que el hombre; todo lo que podemos decir es que hay menos diversidad de función que integrar. Tal criatura obra, como un solo agente, de manera tan verdadera como podemos obrar usted o yo, pero éste es un logro menor, si se considera la variedad de órganos y de actividades posibles. 
No es que los animales sufran más monotonía que nosotros (probablemente sufren menos), sino que sus requisitos para la diversidad "adecuada" son mucho más simples.

Propongo ahora una definición del bien: es el disfrute de los elementos integrados, o armonizados, pero contrastantes de la experiencia, y mientras mayor sea la gama o la riqueza de los contrastes, mayor será el bien, o más alejado estará de la mera trivialidad. Si esto es correcto, entonces la mayor parte de las tradiciones filosóficas han sido incorrectas en parte, y de dos maneras: en primer lugar, han observado demasiado poco que la discordia, sentida como sufrimiento, tiene un mal contrario de monotonia o aburrimiento; y, en segundo lugar, han observado también demasiado poco que, aun admitiéndose el equilibrio entre la unidad y la diversidad, o admitiéndose que se elude tanto la discordia como la monotonía, el resultado todavía puede ser la trivialidad o un bajo grado de valor.

Es correcto que el valor requiere unidad, pero lo que muchos filósofos no parecen haber apreciado debidamente es que la unidad es requerida tanto para los valores más triviales como para los más importantes, y también que la sola unidad no garantiza escapar del aburrimiento, mucho menos que este escape tendrá lugar en un nivel elevado de complejidad, riqueza o intensidad.

Para ser justos con los filósofos más viejos, diremos que su error fue natural, y ello por dos razones cuando menos. La primera es que las condiciones de la vida humana tienden a hacer inevitable una variedad muy grande. El peligro obvio no es la monotonía o un predominio indebido de la unidad y el orden, sino el extremo contrario: el conflicto, la discordia y la anar. quía. Sin embargo, quienes han trabajado en una fábrica o han padecido una mujer o un marido falto de imaginación, esclavo del hábito excesivamente metódico y tedioso, saben cuán grave puede ser el mal de la monotonía. La segunda excusa para el prejuicio filosófico es el problema de la permanencia. La variedad, no considerada dentro de una experiencia, sino entre experiencias sucesivas, parece, en algunos casos cuando menos, inútil y peor que inútil. Si en realidad estamos conscientes del contraste entre el hoy y el ayer, entonces este contraste puede aportar valor al hoy; pero supóngase que olvidamos el ayer, como invariablemente lo olvidamos en cierta medida. O supóngase que estamos muertos y no podemos recordar en modo alguno. Así, la variedad en la forma de impermanencia, el desvanecimiento de las experiencias en el pasado irrecuperable, parece la ausencia del valor, no su presencia. Aquí "los muchos" parecen ser "lo irreal". Ahora existen, ahora no. El budismo fue la primera filosofía que colocó este problema en el centro del pensamiento religioso y filosófico. La impermanencia fue considerada el gran mal. Nada perdura en el mundo de los muchos. De esta suerte, se pensó, debemos buscar la permanencia, o la unidad con respecto al cambio, en lo Uno y no en los muchos, que deben descartarse 
como relativamente irreales. Pero, ¿qué hacer entonces con el hecho obvio de que medimos los grados de valor por niveles de complejidad, y no, como he señalado anteriormente, por el mero logro de la unidad? En todas las grandes tradiciones filosóficas y religiosas existe una tendencia pronunciada, en algunos casos apenas contrarrestada, a descartar o ignorar simplemente la evidencia de la experiencia respecto de este asunto. Yo propongo el procedimiento alternativo, o sea, que consideremos seriamente las implicaciones del hecho de que lo ultra-simple no se tiene por particularmente bueno, pese a su unidad, y que, por lo tanto, debemos buscar otra interpretación de la impermanencia que no sea la de declarar irreal a la pluralidad. En esta búsqueda puede alentarnos la doctrina, profesada algunas veces en el propio budismo, de que la unidad absoluta no está separada de la multitud de fenómenos en el espacio y el tiempo, sino que es "idéntica a ellos". Empero, si la unidad y la pluralidad son simplemente idénticas, entonces lo que es verdadero de la una es verdadero de la otra, y, por consiguiente, si lo Uno es real también lo son los muchos, e igualmente a la inversa. Pero esta doctrina de la identidad es demasiado paradójica, y, ciertamente, si la tomamos al pie de la letra, sencillamente absurda. Y si hay una identidad exacta entre lo Uno unitario y los Muchos infinitamente variados, con sus valores de contraste, ¿qué decir entonces acerca del valor del contraste entre lo Uno y los Muchos? El propio término identidad niega este contraste. Pero el contraste es bueno, y debe ser afirmado. Tanto el contraste entre la unidad, como tal, y la variedad, como tal, cuanto el contraste entre los diversos ejemplos de pluralidad, deben ser considerados positivamente buenos, siempre y cuando puedan ser armonizados o integrados. El problema no consiste en escapar de la pluralidad en el espacio y el tiempo, sino en verla abarcada positivamente, o como real, dentro de una unidad.

Uno puede plantear el asunto de esta manera: si la aparente impermanencia de los fenómenos es real, entonces los fenómenos no pueden ser reales, y los monistas tienen alguna justificación. Pero supóngase que es la impermanencia de los muchos la que es ilusoria. Entonces los mismos muchos pueden considerarse reales sin dificultad. La pluralidad ha sido declarada irreal sobre la base de una supuesta impermanencia; lo que yo deseo argumentar es que esta impermanencia no es esencial a la pluralidad y no es nada más que una ilusión antropomórfica. En verdad, la pluralidad en el tiempo, lejos de requerir impermanencia, requiere permanencia. Si cuando el acontecimiento $B$ sucede, el evento $A$ deja de ser, nunca puede haber una pluralidad consistente en $B$-como-sucesor-de- $A$; hay sólo $B$ con nada que lo suceda o lo siga. San Agustín señaló esto en sus Confesiones, pero en mi opinión no logró en modo alguno extraer la conclusión correcta de su descubrimiento. Su conclusión fue que, desde el punto de vista superior, el de Dios, todos los momentos del tiempo se ven juntos en un solo presente eter- 
no. Pero esto, como dice Bergson, "espacializa el tiempo", hace a la pluralidad en el tiempo esencialmente igual a la pluralidad en el espacio. La totalidad de los acontecimientos es desparramada, de una vez por todas, tanto en el tiempo como en el espacio. Pero esto es la negación del tiempo y el proceso, no su interpretación. Si el todo de las cosas cambiantes es una totalidad no cambiante, entonces nada cambia realmente en modo alguno. Si cualquier cosa viene a ser genuinamente, entonces la totalidad viene a ser, y si hay nuevas cosas cada momento, hay una nueva totalidad cada momento, y una sucesión de totalidades. Pues cada nueva parte añadida al todo hace un nuevo todo.

Verbalmente, las siguientes teorías del tiempo y los acontecimientos son posibles:

1) El acontecimiento presente es real, los acontecimientos pasados y futuros son irreales.

2) Ningún acontecimiento es real, el tiempo es apariencia o ilusión.

3) Todos los acontecimientos, a través del tiempo, son reales; hay una totalidad final de acontecimientos que existen de una vez por todas, a los cuales nunca se añade nada ni se resta nada.

4) Los acontecimientos son reales hasta el presente e incluyendo a éste; los "acontecimientos futuros" no son reales, sino meras posibilidades o probabilidades de ciertos tipos de sucesos. Con cada nuevo acontecimiento se produce una nueva totalidad de acontecimientos, y cada totalidad está incluida en la siguiente. De esta suerte, la expresión "todos los acontecimientos" no tiene significado fijo, sino que se refiere a un todo mayor cada vez que es usada. Según esta concepción, la realidad misma está creciendo, y no hay punto de vista válido más allá de este crecimiento. El conocimiento de la realidad debe crecer con la realidad que está por conocerse, pues conocer un acontecimiento antes de que sucediera sería, según esta concepción, ilusión y no conocimiento. Los acontecimientos no están presentes para ser conocidos sino hasta que suceden.

Deseo argumentar que, de estas cuatro concepciones, sólo la cuarta resistirá la crítica. La primera concepción es un absurdo, pues si los acontecimientos pasados y futuros no son reales, entonces no hay más que un acontecimiento, el presente, y no hay nada que constituya su pasado o su futuro. No sigue a nada, pues no hay nada que seguir. Y no antecede a nada. Según esta concepción, no hay sucesión ni cambio, pues para ello son necesarios cuando menos dos acontecimientos o estados sucesivos.

En cuanto a la segunda concepción, declarar todos los contecimientos "irreales" no constituye ninguna explicación de nuestra experiencia, pues tanto hay una sucesión de apariencias como una apariencia de sucesión. ¿Y apariencia de qué a qué? $\mathrm{La}$ apariencia es una relación y requiere más de un término. $\mathrm{Y}$, además, declarar el cambio irreal es declarar toda búsqueda del 
valor, aun del valor de conocer lo eterno, irreal, pues el logro de este conocimiento es en sí mismo un acontecimiento que el monista trata de producir. Después que éste ha dicho que los acontecimientos no son más que apariencias, continúa presentando la apariencia del intento de controlar el curso de los acontecimientos, eliminando aquellos en los cuales hay creencia en la realidad de los acontecimientos, y produciendo acontecimientos en los cuales no hay creencia en esta realidad, o creencia en lo eterno. Además, la palabra "apariencia" se usa erróneamente en la doctrina. Una apariencia es una entidad real erróneamente tomada por una segunda entidad real como una tercera entidad real: por ejemplo, una reflexión real de árboles que alguien toma erróneamente por árboles. Donde se niega la pluralidad misma, el término "apariencia" pierde todo significado. Sólo podría haber el absoluto que se toma a sí mismo erróneamente por el absoluto.

Llegamos a la tercera concepción: todos los acontecimientos, pasados o futuros, son reales. Declarar asi a todos los acontecimientos partes reales de una totalidad última, a la que no puedé añadírsele ni sustraérsele nada, es tratar los acontecimientos no como sucesos o devenires, sino como meros seres, pues un suceso o devenir es, por su propio significado, una adición a la realidad, algo nuevo, algo que "viene a ser". Pero hasta que $X$ llega a ser, ninguna totalidad que contenga a $X$ puede tener ser. $O$, asimismo, si no hay ni creación ni destrucción dentro de la totalidad de acontecimientos, entonces, o bien no hay ni cambio de devenir en modo alguno, o bien no hay más que un cambio o suceso, el devenir de la totalidad misma. Pero así sustituimos los sucesos reales de nuestra experiencia por un super-suceso mítico, y negamos la realidad de la sucesión, o de los sucesos en cuanto se suceden los unos a los otros, en cuanto vienen a ser, no todos al mismo tiempo, sino uno a uno. Esta concepción es realmente indistinguible de la. segunda, la teoría de que los sucesos son irreales, de que el devenir es ilusión.

Sólo queda la cuarta concepción, la de que los sucesos se crean pero no se destruyen, de que son adiciones inmortales a la realidad, que crece constantemente como un todo. Resulta extraño que esta concepción haya sido tan descuidada. Muchos teólogos han hablado de la creación, pero rara vez parecen haberla tomado seriamente como una categoría básica. Y la filosofía oriental apenas parece haber contemplado la noción de que la permanencia y la novedad real pueden hacerse compatibles, si consideramos el cambio esencialmente como crecimiento o realce de la realidad. Para volver a nuestro concepto del valor en cuanto es medido por la gama de los contrastes armonizados, hay sólo una respuesta a la pregunta: “¿Por qué ha de haber cambio, o de qué sirve el cambio?" Esta respuesta es que el cambio es la adición de nuevas armonías, que, unificándose con lo viejo, producen una mayor armonía total. El logro del valor es, pues, acumulativo, desde el punto de vista cósmico. 
Algunos preguntarán: pero, ¿por qué no ha de existir todo valor eternamente, en lugar de venir a ser paso a paso? La respuesta es que la expresión "todo valor" es ambigua. Si "todo valor" significa todo lo que ahora es actualizado, entonces, puesto que "ahora" tiene un nuevo significado cada vez que se usa, lo mismo sucede con "todo valor", y de esta suerte la realización paso a paso del valor todavía está implícita; si la frase "todo valor" significa todo valor posible, entonces la respuesta es que la actualización de todo valor posible es imposible, puesto que hay valores mutuamente incompatibles. Si uno logra ahora mismo este valor, digamos el de componer un poema, entonces uno no puede lograr ahora mismo ciertos otros valores, por ejemplo el de leer este ensayo con toda atención. Y el universo que lo posee a uno en cuanto tiene uno de esos valores, pasará por alto al que habría poseído el otro valor. Además, la realización absoluta del valor tendría que contener la variedad absoluta, así como la unidad absoluta o perfecta. Pues, una vez más, el valor se mide por la gama de contrastes que son armonizados. Pero "variedad absoluta" es lo mismo que simple caos, y no podría ser unificada. Así, pues, me parece que el supuesto secular de que "lo absoluto" representa el valor supremo es un error. No puede haber un valor real absoluto; más bien, dada cualquier armonía, podría haber uno mayor. La identificación de esta realidad o valor supremos con lo absoluto es la gran ilusión intelectual, una especie de idolatría peculiar de los filósofos y los teólogos. No sólo lo absoluto no es Dios, sino que lo absoluto es apenas un valor o un bien real. Como he argumentado en otro lugar, lo absoluto no es sino un aspecto o elemento abstracto en la deidad; no debe ser adorado como Dios y no es el bien supremo que buscamos. ${ }^{x}$ EI bien supremo es la diversidad armonizada, de la cual no puede haber un máximo final. Por tanto, el devenir es último, pues no habiendo ningún bien que sea el mayor alcanzable, siempre hay algo ulterior que actualizar, como una adición a la armonía que ya es real.

¿Por qué se ha pasado por alto este punto tan generalmente? Es probable que haya varias razones. Pero una de las principales es la tendencia natural a hacer del hombre la medida de las cosas. Para nuestra conciencia humana el cambio aparece, no como pura adición o realce, sino como una mezcla de ganancia y pérdida, pareciendo predominar a veces la pérdida. Sin embargo, el hecho de que la mayor parte del valor del pasado está perdido para nosotros los seres humanos, no significa necesariamente que esté perdido para la realidad en general, como tampoco el hecho de que nuestro conocimiento sea una mezcla de error, ignorancia y "conjeturas más o menos

1 Véase The Divine Relativity, Yale University Press, 1948. Publicado en edición a la rústica en enero de ig63. Capítulo III. Véase también Charles Hartshorne y W. L. Reese, Philosophers Speak of God, The University of Chicago Press, 1952, Introduccion y Ca: pitulo VII. 
cultas" significa que el conocimiento puro, la omnisciencia, no exista en ningún sentido. No todo el pasado está perdido para nosotros, del mismo modo que no toda la realidad nos es desconocida. En la memoria, algo del pasado está todavía con nosotros. Como todas las capacidades humanas, ésta es limitada e imperfecta. Pero la realidad no se mide por nuestras limitaciones. $\mathrm{Y}$ ya hemos visto que el significado mismo de los acontecimientos es el de ser adiciones a la realidad, adiciones que, una vez hechas, son permanentes. Siempre debe ser verdadero que cierto acontecimiento sucedió, ¿y qué puede hacer que esta verdad sea real si no el acontecimiento mismo? De modo que el acontecimiento siempre debe estar ahí, como constituyente real del pasado real. Pero el futuro es otra cosa, pues si fuera real de antemano, no podría haber sucesividad en modo alguno, sino que todo sería al mismo tiempo. La sucesividad tiene lugar entre totalidades de acontecimientos, en que cada acontecimiento nuevo da existencia a una nueva totalidad. La manera de relacionar esto con los modos humanos de conciencia es decir que la memoria humana no es más que una forma imperfecta de la función por medio de la cual la realidad misma conserva su propio pasado, se añade o se resume a medida que procede. La memoria humana no es más que una forma deficiente, del mismo modo que el conocimiento humano es una forma deficiente de conocimiento. La forma superior es divina. $\mathrm{Y}$ aquí tenemos un argumento para aceptar la realidad de Dios: que la verdad misma requiere alguna manera en que la realidad pueda ser acumulativa o creciente, libre de toda pérdida, y que la única clave que tenemos en la experiencia para tal acumulación es la memoria. Así, pues, parece que debe de haber una memoria que no olvida nada, y ésta merecería ser llamada divina.

Puede que ahora se haga claro por qué no podemos aceptar el hinduismo o el budismo, tal como éstos se interpretan usualmente. Estas doctrinas declaran que sólo lo permanente puede ser real, y extraen la conclusión de que los acontecimientos son en cierto modo irreales. ¡Pero los acontecimientos son permanentes! Lo que es ilusorio no es el devenir de los acontecimientos, sino su olvido, la apariencia de que palidecen y se hacen borrosos y nebulosos a medida que se alejan en el pasado. Es para nuestra experiencia que los acontecimientos más remotos se hacen indistintos. Para lo divino, pueden ser perfectamente distintos. Es el futuro el que es nebuloso, y ello no solamente porque nuestra capacidad inferior lo ve poco claramente, sino porque los acontecimientos que todavía no han sucedido no son aún definidos y determinados, siendo posibilidades un tanto indeterminadas. Suceder y hacerse definido son la misma cosa. Ni siquiera Dios puede ver como definido lo que en sí mismo es indefinido.

La depreciación de los particulares concretos tales como "inestable", "perecedero", es pues, creo yo, ilegítima. Lo concreto es absolutamente estable, imperecedero. Pero no es eterno, pues en efecto viene a ser. 
Hay otra razón que explica el descuido o la negación de la concepción acumulativa del cambio. Es lo que yo llamo el prejuicio de la simetría. Los artistas saben que la simetria rígida no es bella, pero los filósofos proceden como si ésta siempre fuera lo que hay que buscar. Así, dígasele a un filósofo que el futuro es irreal, y él probablemente dirá: "Entonces también lo es el pasado." O dígasele que el pasado es irreal, y él dirá: "Entonces también lo es el futuro." Pero lo uno no se sigue de lo otro. Los contrarios no son en general iguales. Los todos no son iguales a las partes, lo complejo no es igual a lo simple, el sujeto no es igual al objeto. ¿Por qué deben ser iguales el pasado y el futuro? Y si lo fueran, entonces el cambio no realizaría nada y sería fútil. El cambio tiene significado sólo si realza la realidad. Uno no puede adoptar el supuesto que hace que el cambio sea absurdo y después proclamar en son de triunfo que uno ha probado este absurdo. Y, sin embargo, eso es lo que han hecho incontables filósofos. Han supuesto lo que se han propuesto probar negando desde un principio la asimetria del tiempo a través de la cual solamente puede el cambio tener significado o servir para el logro de un propósito último inteligible. La dirección del tiempo, la "flecha del tiempo", es precisamente su carácter asimétrico, de acuerdo con el cual sólo son posibles las adiciones, no las sustracciones a la realidad.

Hay otras objeciones al supuesto de la simetría. En general, los conceptoś relacionales básicos son los asimétricos, tales como mayor que, incluido en, posterior a, pues en términos de éstos las ideas simétricas son siempre definibles. Así pues, si ni $X$ ni $Y$ es mayor que la otra, entonces son iguales; pero uno no puede, mediante la negación de la igualdad, indicar cuál es mayor. De esta suerte, los principios elementales del pensamiento son asimétricos, no simétricos. ¡Cuán pocas veces se ha hecho justicia a esta verdad y a sus consecuencias!

Hemos esbozado una teoría del valor o el bien, y mostrado que esta teoría favorece una filosofía del devenir más bien que del mero ser. El valor, el bien, se mide por la suma de contraste o variedad que se armoniza en la experiencia. Por tanto, la variedad de los particulares concretos en el espacio y el tiempo tiene un valor positivo. Ninguna mera unidad podría ser un sustituto de esta variedad. Y tampoco podría haber una variedad absoluta, por encima del espacio y el tiempo, cuya perfección hiciera innecesaria la variedad lograda a través del devenir o el proceso, pues ninguna variedad, sea en el tiempo o no, puede ser absoluta o la mayor posible. Dada cualquier varicdad, siempre puede haber una mayor, del mismo modo que, dado cualquier número, puede haber otro mayor. No podria haber el mayor número posible ni la mayor variedad posible; por tanto, puesto que el valor se mide por la variedad, no es concebible el mayor valor posible. Por consiguiente, el devenir es la única respuesta racional a la naturaleza del bien. 
Siempre puede haber algo mejor; obviamente, entonces, debe haber algo mejor. De aquí el proceso o el devenir.

Hemos visto también que no es correcto objetar al devenir que sus productos son meramente temporales. El olvido humano del pasado no es prueba de que éste ha dejado de ser. Hay un absurdo en la idea de que algo ha sucedido, pero sin embargo ya no es verdad que haya sucedido; $y$, puesto que la verdad es concordancia con la realidad, la verdad de que algo ha sucedido significa la realidad de su haber sucedido, y de aquí la realidad del acontecimiento en la forma de una ocurrencia pasada. La concepción lógica del pasado es, pues, que éste es real y permanente.

El devenir, concluimos, puede aceptarse como perfectamente real, y sus productos como indestructibles. Estos productos son acontecimientos o sucedidos, no cosas, sustancias, personas. Aquí el budismo tenía incluso más razón de la que suponía, en su negación de lo que frecuentemente se ha significado por "sustancia" o "alma". Como lo comprendieron los budistas mucho antes que nadie, la realidad del alma está en sus estados o experiencias momentáneas. Éste fue uno de los más grandes descubrimientos metafísicos, vivificados en el Occidente por Hume, William James y Whitehead. Pero, lamentablemente, este descubrimiento fue viciado, tanto en el budismo como en Hume, por la noción de la impermanencia de los estados y la irrealidad de los particulares en el tiempo y el espacio. Es cierto que, en último término, hay en el budismo una aceptación ambigua de los particulares cuando se dice que éstos son en cierto modo "idénticos" a la realidad absoluta que está más allá del espacio, el tiempo y la pluralidad, y más allá de la concepción racional. Pero esto no basta, pues no sólo son los particulares tan reales como cualquier universal, sino que son superiores a cualquier realidad tal; no sólo es el devenir igual al puro ser, sino que lo sobrepasa infinitamente, pues es el devenir el que contiene sus propios contrastes con el ser, no el ser el que contiene sus contrastes con el devenir. Y el contraste es la medida del valor (admitida la armonía de los contrastes). La prueba de que el devenir se contrasta con el mero ser, pero no el ser con el devenir, es la siguiente.

Si el contraste del ser con cada nueva fase del devenir estuviera en el propio ser, entonces cada acto de devenir debería producir en el ser inmutable un contraste novel. Pero entonces el ser no sería inmutable. Cualquier combinación de lo nuevo y lo viejo está destinada a ser nueva. Por tanto, la novedad es la forma inclusiva de la realidad. Más aún, puesto que ninguna totalidad de acontecimientos puede ser la mayor posible, una totalidad 'eterna de contrastes incluidos dentro del ser significaría darse por satisfechos con un bien menor que el posible. La única solución es el devenir de una nueva totalidad cada momento, por siempre. En cada totalidad pueden estar contenidos tanto el ser inmutable como cualquier contraste dentro de y con 
el ser, pues para formar un nuevo todo no es necesario que todas las partes, factores o constituyentes sean nuevos. Basta con que algunos lo sean. Éste es el principio del devenir concebido como sintesis creativa: el sublime descubrimiento de Whitehead. Para producir novedad no es necesario destruir nada, sino únicamente crear algo. $\mathrm{Y}$ para aceptar el proceso, no hay necesidad de rechazar un elemento inmutable, pues la combinación de una cosa variable y una cosa constante es ella misma una cosa variable. Así, pues, si $V$ es una variable y $C$ es una constante, entonces $V C$, la combinación, es obviamente una variable. Esto, pienso yo, es matemáticamente evidente. De ahí que el devenir, no el ser, sea la idea última u omniincluyente.

Ahora bien, ¿reconoce esto el budismo? Aparentemente no. Después de declarar que los estados momentáneos del alma constituyen su realidad, los pensadores budistas procedieron, ello no obstante, a negar la plena realidad de esos estados, y de todo devenir y particularidad en el tiempo y el espacio. En otras palabras, después de decir que los estados o experiencias son más últimos que la identidad ${ }^{\circ}$ la unidad del alma, invirtieron la dirección de su pensamiento y declararon que alguna unidad o identidad, o en todo caso la no-pluralidad, inmune al devenir, es cuando menos tan real o última como los estados. Algún bien sereno, a salvo de todo el peligro, el conflicto y el sufrimiento a que están expuestos los individuos capaces de entrar en conflictos en deseos y propósitos debido a sus relaciones en el tiempo y el espacio, es la verdadera realidad. No tenemos que ver en esto un mero error; pero yo me aventuro a pensar que están implicados uno o más errores. Pienso también que hay una unidad superior a cualquier realidad humana ordinaria. Pero no es una unidad más allá de todo devenir, sino más bien la unidad del devenir mediante la cual los acontecimientos, una vez creados, están indestructiblemente presentes en la realidad de ahí en adelante. Lo que es ilusorio no es la creación, y el devenir como síntesis creativa, sino la noción de que los productos pasados de este proceso creativo están perdidos, a medida que el proceso continúa.

Debe admitirse que los productos pasados del devenir se pierden en gran medida en cuanto se refiere a la conciencia humana. Tales productos se pierden para nosotros, nosotros ya no los disfrutamos ni los poseemos. Pero esto no prueba que estén perdidos para la realidad cósmica, del mismo modo que nuestra ignorancia sobre otros planetas no prueba que no ocurra gran cosa en ellos. $\mathrm{Y}$ aun en nosotros hay alguna posesión de acontecimientos pasados, a través de nuestras memorias. Todo lo que tenemos que hacer para concebir la plena posesión del pasado es concebir una forma perfecta de memoria, una forma que no esté mezclada en modo alguno con el olvido. Ésta es la idea de una memoria divina. $\mathrm{Y}$ aquí tenemos un argumento para creer en Dios: el argumento de que sólo así podemos hacer inteligible lo que se significa por devenir. Pues, como hemos visto, el devenir no puede ser la 
destrucción de los acontecimientos, puesto que es una contradicción decir: "Algo realmente sucedió, pero no hay una realidad de este suceso pasado." Si el suceder pasado no fuera nada ahora, entonces la verdad acerca de él sería igual que la verdad acerca de nada. Pero éste no es el caso. Además, los budistas mismos siempre están hablando sobre cómo lo que uno ha hecho en el pasado influye en el presente. Sin embargo, la "influencia del pasado en el presente" es sólo un nombre para el problema de la permanencia. Nuestra única clave positiva para una relación real de la experiencia presente con el pasado, se encuentra en la memoria. Puesto que la memoria humana es incompleta, la verdadera medida de la permanencia debe ser una memoria completa.

Charles Hartshorne

Universidad de Texas

(traducción de José Luis González) 